\title{
Does the use of implanters affect the quality of FUE grafts?
}

\author{
Conradin von Albertini, MD I Zurich, Switzerland I info@swisshairdoctor.ch; \\ Marie-Anne von Albertini, PhD I Zurich, Switzerland
}

\section{INTRODUCTION}

Until recently, implanter pens were the Sleeping Beauty of hair restoration surgery. After Choi introduced the placing devices more than 25 years ago, only a few trod the thorny path toward using them. ${ }^{1,2}$ Not only is placing grafts with implanters trickier than with forceps, but it also increases the workload for the surgeon. The drawbacks of implanters seemed to offset their supposed beauty - that is, causing less graft damage during placing than forceps. In many clinics, the pens landed in the famous "closet for useless surgical gadgets," waiting there for years to be awakened.

Nevertheless, with the portion of FUE procedures growing, the interest in implanters has surged. Because FUE grafts are more frail and smaller than FUT grafts, even seasoned teams struggle with placing them. Many surgeons have therefore turned to implanters to prevent the frequent crushing and hooking of FUE grafts. ${ }^{3-6}$ The option to pre-make the incision and delegate placing with flexible $^{3,7}$ or dull needle ${ }^{4}$ implanters to technicians has further increased the popularity of the device.

Amid the new hype, however, concern is growing that implanters cause their own set of complications. ${ }^{8}$ The allegations vary, but they mostly fall into two broad categories:

1. Loading issues: damage caused by inserting the grafts into the pen. A typical worry is that grafts suffer "needle squeezing," a crushing injury that occurs when grafts are pressed into the groove of the implanter needle. Another concern is that teams encounter difficulties with loading and can injure the grafts.

2. Placing issues: growth failures caused by planting grafts with the device due to, for example, implanters causing grafts to hook or break on a regular basis.

Unfortunately, with scant scientific data available, the discussion about the benefits and limitations of implanters remains mostly anecdotal. For instance, only limited evidence supports the claim that implanters are less traumatic to the grafts than forceps. Early studies on the survival of 1- and 2-hair follicles in Asian patients suggested that implanters increase the chances of graft survival. ${ }^{9,10}$ However, similar results may not be seen with larger 3-hair grafts or in Caucasians. ${ }^{8}$ Existing research on graft trauma and graft quality is not helpful either because it is based on or assumes placing by forceps rather than implanters. ${ }^{11-14}$

\section{OBJECTIVES}

The goal of this study was to provide new evidence on graft quality after the use of implanters. The study addressed the following questions:

1. Are there graft injuries, such as follicle crushing, paring, transection, hooking, or breakage, that appear after placing with implanters and were not present before placing the graft?
2. Do difficulties regularly arise with the use of implanters, for example, a long time out of solution (TOS) or multiple attempts to load implanters?

The study results will enrich current discussion about the benefits of using implanters when placing FUE grafts with particular emphasis on the practical implications of the findings.

\section{MATERIALS AND METHODS}

This in-depth study was based upon FUE procedures performed on the frontal region of seven Caucasian men. In all cases, the donor and the recipient hairs were fully trimmed to a length of $1 \mathrm{~mm}$. A total of 15,147 grafts were transplanted, with an average of 2,009 grafts per patient (Table 1).

\begin{tabular}{|c|c|c|c|c|c|c|c|}
\hline$C=$ & 蓶 & Trifh & 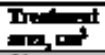 & 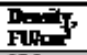 & OPL & 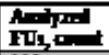 & 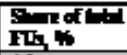 \\
\hline 1 & 25 & 2,388 & 64 & 37.3 & $8: 15$ & 100 & 4.2 \\
\hline$\overline{\mathbf{2}}$ & 75 & 2,56 & 85 & 353 & 855 & 50 & 39 \\
\hline 3 & 52 & 2,214 & 58 & 38.2 & 8.55 & 95 & 4.3 \\
\hline 4 & 35 & 2.191 & 56 & FI & $7 \mathbf{3 x}$ & S4 & 25 \\
\hline 5 & 40 & 2,187 & 56 & 39.1 & 8.20 & 56 & 2.6 \\
\hline 6 & $\mathbf{7 6}$ & 215 & 8 & 353 & 87 & 56 & 26 \\
\hline 7 & 39 & 1,741 & 50 & 34.8 & $7: 00$ & 31 & 1.8 \\
\hline Th & & Epla & 80 & & & 81 & \\
\hline Average & 35 & 2,009 & 58 & 37.2 & $8: 05$ & 69 & 3.2 \\
\hline
\end{tabular}

Extraction was performed with a motorized sharp serrated punch, and sharp needle implanters were used for placing without premade incisions. Extraction and implantation were done in multiple sweeps to ensure a time out of the body of less than 2 hours. The grafts were not split or trimmed. A physician performed the extraction and the implantation. Both he and his team are experienced implanter users $(>1,000,000$ FUs within 7 years). Two separate approaches were used to assess the quality of the grafts and the implanting process:

1. Analysis of the graft quality before and after implantation. The study included 1,292 hair follicles (hairs) on 482 grafts (FUs), accounting for $3.2 \%$ of the 15,147 planted FUs. The grafts were chosen randomly. We analyzed an average of $69 \mathrm{FUs}$ (31 to 100) before and after implantation in each case. First, we photographed each FU under $10 \times$ magnification and from various angles. Next, each FU was placed with an implanter, directly re-extracted, and photographed again. We assessed the pictures of the grafts before and after implantation. Finally, we conducted data analysis and statistical modeling.

2. Analysis of the quality of the implanting process. The loading by two technicians and the placing by the physician were video-documented for approximately 10 minutes. We examined the sequences for the implantation speed, the number of loading attempts, and the TOS (i.e., how long it takes from the moment a graft is seized for loading until it is placed). Loading and placing were also scrutinized for unexpected issues. 


\section{RESULTS}

Both analyses support the claim that implanters protect denudated FUE grafts.

\section{Graft quality before and after implantation}

The data indicate that implanters leave grafts intact. After implantation, only 6 hair follicles $(0.5 \%)$ displayed damage that was not previously present, and $99.5 \%$ of the hair follicles remained unchanged (Table 2). In short, implanters have a negligible impact on the quality of grafts, which was confirmed in statistical analysis. In an ANOVA test, the use of implanters did not significantly affect the graft quality $\left(\mathrm{F}_{1,962}\right.$ with $p$-value 0.73$)$. Furthermore, the graft quality did not differ significantly between patients $\left(F_{6,956}\right.$ with $p$-value 0.17$)$.

TABLE 2. Hair counts and damage assessment

\begin{tabular}{|c|c|c|c|c|c|c|c|c|c|}
\hline & 1 & Fon & Trioned & عـ & Finder & Ereld & Darided & تحسب & S \\
\hline Before & 1,292 & 49 & 54 & 2 & F & - & [- & 105 & \\
\hline In \% & $100 \%$ & $3.8 \%$ & $4.2 \%$ & 0.286 & - & - & - & $8.7 \%$ & \\
\hline After & 1,292 & 49 & 54 & 4 & 3 & - & 1 & 111 & +6 \\
\hline$x=\pi$ & $\sin 6$ & $90 \%$ & 124 & $a=1$ & 220 & $=$ & $a \sqrt{x}$ & 200 & $+25 x$ \\
\hline
\end{tabular}

It is important to note that some hair follicles had preexisting damage. About $8.1 \%$ of the hair follicles presented with transection, paring, or breakage prior to implantation.

After placement, the rate of damaged hairs increased to $8.6 \%$. Remarkably, none of the hairs displayed any crush injuries. Moreover, the device did not pare or transect any of the grafts; however, hooking occurred in rare cases: 3 previously intact hair follicles $(0.3 \%)$ were hooked after implantation and $2(0.2 \%)$ were broken, possibly as a result of hooking. Furthermore, 1 follicle $(<0.1 \%)$ presented with an unexpected, implanter-specific injury, which we dubbed "bulb decapitaFIGURE 1. Intact graft before implantation (A) and
with a bulb decapitation after implantation (B)
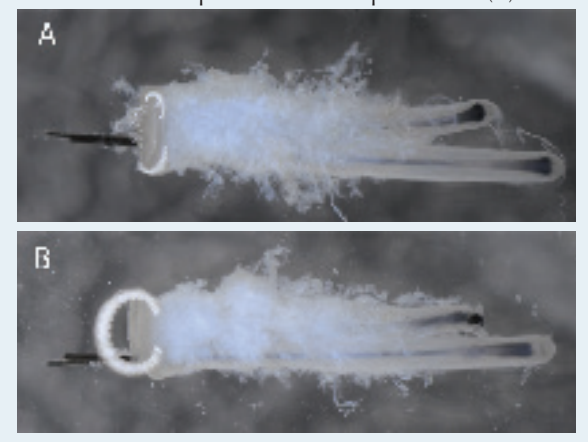

tion" (Figure 1).

\section{Quality of the implanting process}

The videos recorded the implantation of 150 grafts in 590 seconds and showed a smooth process without systematic issues. With an average of 1,019 grafts per hour, the implan-

tation speed was high. Moreover, loaders needed to reload a graft only once, accounting for $0.6 \%$ of the observed implantations (Table 3). On average, the TOS lasted 8.2 seconds.

More surprising are the insights into how implanters cause hooking (Figure 2). Hooking occurs when one of the bulbs protrudes from the needle's groove. As the loaded needle enters the skin, the bulb remains stuck on the surface.
Eventually, the follicle folds into what is best described as a "distal hook" as it affects the upper portion of the follicle.

While splayed and large follicles are at risk for proximal hooking, the video revealed another culprit: trimmed hair growing around the recipient sites. Like little spears, the hairs enter the groove of the approaching implanter needle, lift a bulb, and cause a hook (Figure 3).

\section{DISCUSSION}

The evidence suggests that implanters protect the integrity of denudated FUE grafts. They leave the grafts intact, with no visible loading injuries, and placing injuries are extremely rare. This outcome has several interesting implications for the implanter practice.

\section{Loading injuries are absent, even in untrimmed grafts of multiple follicles}

One of the most surprising results of this study is the complete absence of visible loading injuries.

Most importantly, we found no indications of needle squeezing. If needle squeezing had occurred, it should translate into at least some crushing injuries, which, however, were also entirely absent. No other damage attributable to the event was visible.

This outcome refutes the hypothesis that grafts are damaged because they are "pressed" into the needle's groove. Intriguingly, the FUs were relatively large (Table 4), which could have increased the risk for needle squeezing. The average hair/FU ratio was 2.7 , whereas $61.2 \%$ of the FUs had 3 or more follicles. The transection rate (as \% of expected hairs) was $4 \%$. None of the FUs were split or trimmed. Moreover, Table 4 shows that in 5 of the 7 cases, the implanter sizes were smaller than the punch sizes $(\Delta=-0.05 \mathrm{~mm}$ to $-0.20 \mathrm{~mm})$, which counters the notion that the implanter and the punch need to be matched by size to avoid needle squeezing.

Although the findings seem counterintuitive, it is impor-

\begin{tabular}{|c|c|c|c|c|c|c|c|}
\hline II & $\begin{array}{l}\text { Primo, } \\
3\end{array}$ & I!! & & & 5an: & $\operatorname{Tos} ; 1$ & 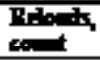 \\
\hline 7 & 280 & 84 & 0.30 & 18.0 & 1080 & 8.3 & 1 \\
\hline a & 310 & 8 & 057 & 161 & 954 & $\mathbf{8 1}$ & 0 \\
\hline Totals & 590 & 167 & & & & & \\
\hline Alsis & & & $\mathbf{L}$ & $I 7$ & गए & 27 & $\overline{\Delta x}$ \\
\hline
\end{tabular}
tant to remember that FUs were extracted with a depth-controlled sharp punch. Thus, the punch defines the diameter of the graft only until a scoring depth of approximately $2.5 \mathrm{~mm}$. No further dissection occurs at a greater depth because the FUs are de-tethered with forceps, a process that strips the lower portion of the 
TABLE 4. Graft size parameters, punch and implanter diameters

\begin{tabular}{|c|c|c|c|c|c|c|c|c|c|c|c|}
\hline I1) & Py & $\begin{array}{l}\text { Anthil } \\
\text { rins }\end{array}$ & $\begin{array}{l}\text { Tringfing } \\
\text { Fins }\end{array}$ & Iryed & Fu's & FDs & $\begin{array}{l}\text { Fos af } \\
3-5\end{array}$ & 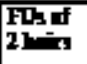 & Pin & ifici, & \\
\hline 1 & 273 & 259 & 13 & $4.8 \%$ & 2.6 & 100 & 55 & 45 & 1.00 & 0.8 & -0.20 \\
\hline $\mathbf{2}$ & 244 & 76 & 7 & $29 \%$ & 26 & 90 & 57 & $\mathbf{3}$ & Eक & 01 & -15 \\
\hline 3 & 275 & 267 & 5 & $1.8 \%$ & 2.8 & 95 & 69 & 26 & 1.00 & 1.0 & - \\
\hline 4 & 101 & 151 & 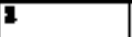 & $50 \%$ & 28 & 54 & $\mathbf{n}$ & n & 1.18 & $1 E$ & -11 \\
\hline 5 & 140 & 129 & 11 & $79 \%$ & 23 & 56 & 16 & 40 & 1.05 & 1.0 & -0.05 \\
\hline 6 & 15? & 194 & 3 & $19 \%$ & 28 & $\mathbf{5 0}$ & 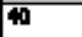 & 16 & 1.00 & $1 \boxminus$ & - \\
\hline 7 & 99 & 96 & 3 & $3.0 \%$ & 3.1 & 31 & 26 & 5 & 1.05 & 1.0 & -0.05 \\
\hline$\theta$ & 1E: & $1 \dot{x}$ & $=$ & $\mathrm{IO}$ & $2 y$ & $\operatorname{lin}_{12}$ & $\begin{array}{l}2: 5 \\
617 x\end{array}$ & $\begin{array}{l}15.7 \\
38,5 x\end{array}$ & & & \\
\hline
\end{tabular}

${ }^{1}$ Number of follicles before transection; ${ }^{2}$ Transected hairs/expected hairs

follicles from their surrounding tissues. As a result, the bare follicles are smaller than their epithelial plugs. Even large and untrimmed follicles fit comfortably into the needlewith their epithelial plugs resting outside of the groove (Figure 4).

FIGURE 4. Loaded graft with epithelial plug resting outside of the needle's groove

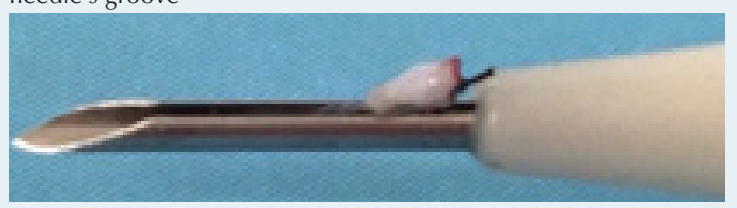

In other words, needle squeezing is a nonissue for denudated FUE grafts.

Reducing the size of the grafts or matching the diameters of the needle and the punch are rarely necessary. Surgeons should choose the implanter needle to fit the follicles, not the epithelial plugs.

Less surprising is the finding that no regular issues occurred during the loading process. In a team with precise loading skills, the TOS lasts only seconds. Multiple loading attempts are an exception. However, practice is necessary, and physicians need to train their teams properly with small trials before using implanters in larger procedures.

\section{Placing injuries are a rarity-distal hooking and bulb decapitation revealed}

The claim that implanters are atraumatic to the grafts mostly refers to their ability to place follicles without crushing or hooking, two complications of placing FUE grafts with forceps. The assertion is that the implanter needle acts like a splint, which allows the follicles to be inserted into the recipient site without direct pressure on the bulb and

FIGURE 5. Graft before (A) and after (B) implanter use
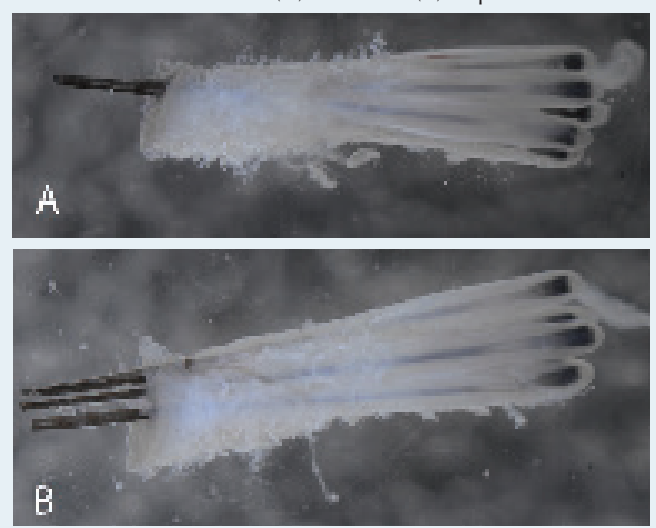

without folding or distorting the follicle.

The evidence of intact hair follicles in this study overwhelmingly supports the contention that implanters avert placing injuries of FUE grafts (Figure 5).
Most importantly, implanters successfully prevent crush injuries to denudated follicles. None of the grafts presented with crushing damage after implantation. However, in contrast to common belief, implanters do not entirely prevent hooking because-in extremely rare instances - they cause distal hooking. The better known "proximal hooking" occurs when forceps fold the lower portion of the follicle deep inside the incision, creating a small hook near the bulb, which remains hidden until the graft eventually fails to grow. In contrast, distal hooking affects a higher portion of the follicle and happens in plain sight. It occurs when a bulb remains stuck on the surface as the implanter is inserted, eventually folding the follicle (Figure 3). Distal hooking is immediately visible and, more importantly, it is instantly rectifiable, which probably explains why the damage affected only a few hairs in this study.

Because the placer needs to recognize and address distal hooking as it happens, a lack of experience is a risk factor, especially with closely trimmed but hairy recipient sites or grafts with splayed follicles. Physicians, particularly those delegating the placing with dull needle implanters to technicians, should be aware of this issue.

The other implanter-specific injury revealed in this study was bulb decapitation, which occurs when a bulb protrudes from the anterior opening at the needle's tip. As the needle enters the skin, the bulb slides underneath the sharp tip, which then slices off the bulb. Fortunately, this damage affected less than $0.1 \%$ of the hairs in this study. Nevertheless, surgeons need to be aware of the risk, especially when grafts are large. A placer should always check the loaded needles for "low-lying" bulbs and, if necessary, return the device to the team to reload it properly.

\section{Focus on planting denudated FUE grafts-more research needed}

The findings suggest that placing with implanters protects denudated grafts. However, more research is necessary for a full picture. First, we did not compare implanters with forceps; therefore, endorsing one method over the other is not possible. Second, the outcomes apply to sharp needle implanters and may not be entirely transferable to devices with dull or flexible needles. Third, we analyzed only denudated grafts extracted with a sharp punch. It remains to be seen if the findings also apply to the chubbier grafts resulting from hybrid or blunt punch extractions or even to FUT grafts. Fourth, while unlikely, the re-extraction of the grafts could have led to injuries unrelated to the device. Finally, the 10x magnification in this study is not sufficient to exclude injuries on the cellular level.

\section{CONCLUSION}

Sharp needle implanters seem to be safe and efficient for placing denudated FUE grafts obtained by a sharp punch. We found no indications that grafts suffer from loading and that graft damage from placing was nearly nonexistent. The device can indeed cause two rare injuries, distal hooking and bulb decapitation, but with sufficient experience and caution of the physician and team, the risk is negligible. Moreover, the findings lead to some useful hints for implanter practice: 


\section{Loading implanters:}

- Damage from "needle squeezing" is unlikely with denudated grafts - the choice of the implanter needle should be dictated by the dimensions of the proximal follicles, not the punch size.

- Multiple loading attempts or slow speed are a sign of insufficient experience-loaders should practice in smaller cases before using the pens in larger procedures.

\section{Placing with implanters:}

- Implanters prevent crushing and most of the hooking when placing FUE grafts-in experienced hands, the risk of "distal hooking" and "bulb decapitations" is marginal.

- Risk factors for distal hooking are splayed follicles and shaved, but hairy recipient sites-the placer needs sufficient practice to recognize and immediately rectify hooking.

- Bulb decapitation is more likely to occur with large and splayed follicles - the placer has to watch for "lowlying" bulbs and if necessary return the implanters for reloading straightaway.

In summary, even if it is too soon to tell the end of the story, implanters appear beautiful enough to be awakened by the kiss of denudated FUE grafts. Time shall tell if they will live happily ever after.

\section{References}

1. Lorenzo, J., and X. Vila. Introduction to the use of implanters. Hair Transplant Forum Int'I. 2011; 21(4):121-122.

2. Choi, Y.C., and J.C. Kim. Single hair transplantation using Choi hair transplanter. J Dermatol Surg. 1992; 18(11)945-948.
3. Shiao, T.K., and I.S. Shiao. Easier and more efficient implanting: a two-handed technique for faster and less traumatic graft placement. Hair Transplant Forum Int'I. 2006; 16(5):169-170.

4. Speranzini, M. FUE Graft placement with dull needle implanters into premade sites. Hair Transplant Forum Int'I. 2016; 26(2):49, 54-56.

5. Rassmann, W. The future of hair transplantation. Hair Transplant Forum Int'l. 2015; 25(5):198-199.

6. Knudsen, R. Controversies: Forceps vs. implanters. Hair Transplant Forum Int'l. 2014; 24(2):57-58.

7. Shiao, T.K., J.C. Shiao, and I.S. Shiao. Hair Sciences: Shiao needle sleeve: a new depth control sleeve that fits different sized needles for creating recipient sites. Hair Transplant Forum Int'l. 2009; 19(3):88-90.

8. Ahmad, M., and M.H. Mohmand. Limitation of hair implanters: another perspective. Hair Transplant Forum Int'l. 2016; 26(3):111-112.

9. Lee, S.J., et al. Evaluation of survival rate after follicular unit transplantation using the KNU Implanter. Dermatol Surg. 2001; 27(8):716-720.

10. Lee, W., et al. Survival rate according to grafted density of Korean one-hair follicular units with a hair transplant implanter: experience with four patients. Dermatol Surg. 2006; 32(6):815-818.

11. Devroye, J. Improving Graft Survival: Extraction Techniques and Transection Management. In: S.M. Lam and K.L. Williams, eds. Hair Transplant 360: Follicular Unit Extraction, Vol 4. New Dehli: Jaypee Brothers, Medical Publishers Pvt. Limited; 2015:133-150.

12. Gandelman, M., et al. Light and electron microscopic analysis of controlled injury to follicular unit grafts. Dermatol Surg. 2000; 26(1):25-31.

13. Cooley, J.E. Optimal graft growth. Facial Plastic Surgery Clinics of North America. 2013; 21(3):449-455.

14. Parsley, W.M., and D. Perez-Meza. Review of factors affecting the growth and survival of follicular grafts. Journal of Cutaneous and Aesthetic Surgery. 2010; 3(2):69-75.

\section{Wireless Follicular Dermatoscope}

\section{Wirelessly Capture Pictures:}

Twelve distinct levels of polarization. Observes and records in real time (30 FPS). Transmits within a range up to $20 \mathrm{ft}$. Built-in snapshot button. 126-FS-1

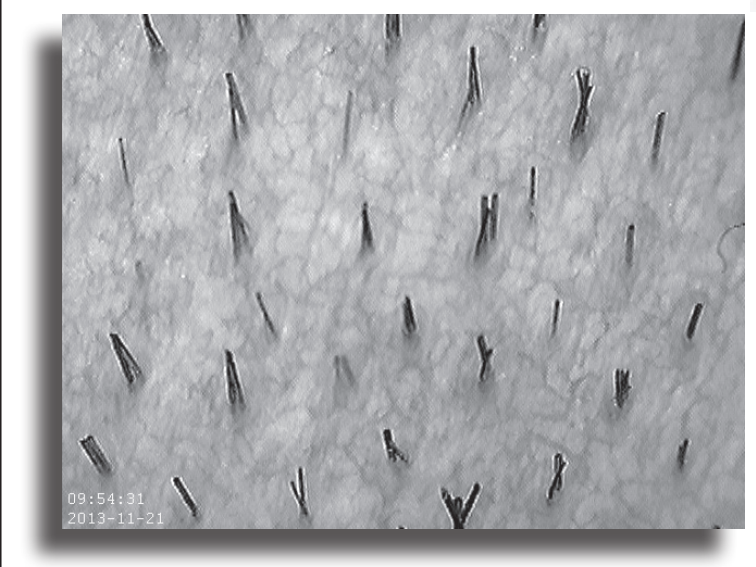

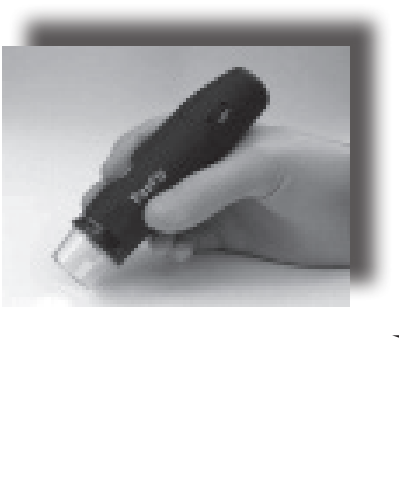

\section{$\$ 349.00^{*}$}

*When you mention this Forum ad.

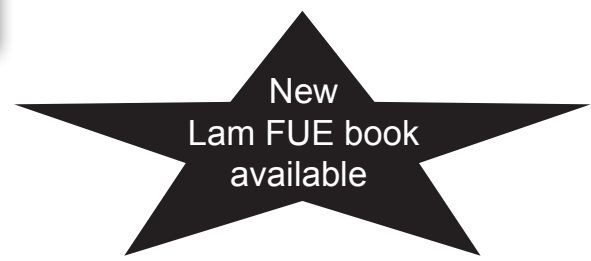

